\title{
The Novel Circular RNA Circ-PGAP3 Promotes the Proliferation and Invasion of Triple Negative Breast Cancer by Regulating the miR-330-3p/Myc Axis
}

\author{
Dabao $\mathrm{He}^{\prime}$ \\ Xiaoling Yang ${ }^{2}$ \\ Wenbin Kuang' \\ Guoqing Huang' \\ Xiaohong Liu' \\ Yonggang Zhang' \\ 'Department of Laboratory Medicine, \\ Shenzhen Longhua District Central \\ Hospital, Shenzhen 518110, People's \\ Republic of China; ${ }^{2}$ Department of \\ Laboratory Medicine, Shenzhen Baoan \\ District Songgang People's Hospital, \\ Shenzhen 518105, People's Republic of \\ China
}

This article was published in the following Dove Press journal: OncoTargets and Therapy

Background: Triple negative breast cancer (TNBC), a special subtype of breast cancer, is characterized by high recurrence, mortality and few treatments. To date, the key factors contributing to TNBC progression have not been fully identified. In the current study, we found a TNBC-related circular RNA (circRNA), circ-PGAP3, and explored its biological function, clinical significance and potential mechanism of action.

Materials and Methods: The functional assay was carried out using CCK-8, colony formation and Transwell invasion assays. RIP, RNA pull-down and luciferase reporter assays were used to test the correlation between circ-PGAP3, miR-330-3p and Myc. The animal model was employed to verify the function of circ-PGAP3 in vivo.

Results: Circ-PGAP3 expression was significantly increased in TNBC tissues. High circPGAP3 was closely associated with large tumor size, lymph node metastasis, later TNM stage and dismal outcome. Through performing a series of in vitro and in vivo experiments, we found that circ-PGAP3 promoted TNBC cell growth and metastasis via sponging and inhibiting miR-330-3p, resulting in the upregulation of proto-oncogene Myc. Importantly, circ-PGAP3 expression was positively correlated with the Myc protein level but negatively correlated with miR-330-3p expression in TNBC tissues. Moreover, silencing of miR-330-3p or overexpression of Myc could effectively rescue the weakened malignant phenotype induced by circ-PGAP3 knockdown.

Conclusion: Our results unveil the important driving role of circ-PGAP3 in TNBC development and progression, which provides a candidate therapeutic target for TNBC patients.

Keywords: triple negative breast cancer, circular RNA, miRNA, Myc

\section{Introduction}

Triple negative breast cancer (TNBC) refers to breast cancer with negative estrogen receptor, progesterone receptor and human epidermal growth factor receptor 2, accounting for $12 \%$ to $17 \%$ of all breast cancers. ${ }^{1}$ TNBC is highly aggressive and prone to local recurrence and distant metastasis. ${ }^{2,3}$ Compared with the 5-year survival rate of more than $90 \%$ for other types of early-stage breast cancer, the 5 -year survival rate of early-stage TNBC is only $77 \%$ and that of later-stage breast cancer is only $14 \% .{ }^{4}$ Due to the lack of effective therapeutic targets for TNBC, the current treatments are very few, mainly chemotherapy. ${ }^{5}$ Although chemotherapy can prolong the survival time of TNBC patients to some extent, its effect is
Correspondence: Xiaoling Yang Department Shenzhen Baoan District Songgang People's Hospital, Shenzhen 518105, People's Republic of China Tel +86 0755-27718530

Email xly_szbasg@yeah.net 
relatively limited, and high-intensity chemotherapy will have a great impact on the quality of life of patients. ${ }^{6}$ Therefore, it is urgently needed to identify more effective candidate targets to antagonize this fatal disease.

Research interest has recently been re-ignited in the field of non-coding RNA, especially circular RNA (circRNA), a covalently closed endogenous RNA produced by a back-splicing process. ${ }^{7}$ CircRNA has no 3-terminal cap structure and 5-terminal poly-A tail, enabling it to be free from digestion by RNA exonuclease and very stable. ${ }^{8}$ With the widespread application of RNA sequencing technology and the rapid development of biophysical technology, it was found that many exons can undergo non-linear reverse splicing or gene rearrangement to form circRNA, accounting for a large proportion in all splicing transcripts. ${ }^{9}$ The current research on the function of circRNA shows that it mainly plays a role via the following four models: acting as a miRNA sponge, regulation of gene transcription, binding to functional protein and even translation of protein. ${ }^{10}$

In recent years, an increasing number of studies have shown that circRNA is frequently abnormally expressed in various human cancer types, and it functions as a fundamental player in tumorigenesis and development through direct or indirect ways. ${ }^{11-13}$ However, the molecular function and biological role of circRNA in TNBC remain largely unknown. In a previous study, Zeng et al performed circRNA high-throughput sequencing in paired TNBC and normal tissues, and found a large number of dysregulated circRNAs, including circ-PGAP3 (hsa_circ_0106800, chr17: 37843549-37844086). ${ }^{14}$ Here, we explored the expression level, clinical implication and biological function of circ-PGAP3 in TNBC. Moreover, the underlying mechanism accounting for the pro-tumor effect of circ-PGAP3 was also illustrated.

\section{Materials and Methods}

\section{TNBC Tissues and Ethical Approval}

We collected 86 paired TNBC and normal tissues from Shenzhen Longhua District Central Hospital to test circPGAP3, miR-330-3p and Myc expression. All patients were diagnosed with TNBC and had not received any antitumor therapy. The clinicopathological characteristics of enrolled patients were obtained, and their relationships with circ-PGAP3 were analyzed. The informed consent was obtained from each patient and this study was approved by the ethical standards of the Ethics
Committee of Shenzhen Longhua District Central Hospital in accordance with the Declaration of Helsinki.

\section{RNA Isolation and qRT-PCR}

Total RNA was regularly extracted by TRIzol reagent (Invitrogen, CA, USA) with the standard protocol, and the cDNA was synthesized using the PrimeScript ${ }^{\mathrm{TM}}$ Reverse Transcriptase kit (Takara, Shiga, Japan) with random primers. Then, cDNA was amplified and quantified in triplicate using ChamQ ${ }^{\mathrm{TM}} \mathrm{SYBR}^{@}$ qPCR Master Mix (Vazyme Biotech, Nanjing, China). The housekeeping gene GAPDH was used as the reference control and the control group was considered $100 \%$ expression. The $2^{-\Delta \Delta \mathrm{Ct}}$ method was applied to assess relative gene expression.

\section{Subcellular Localization}

TNBC cells were collected and then treated with the Cytoplasmic \& Nuclear RNA Purification Kit (Norgen Biotek, ON, Canada) to extract cytoplasmic and nuclear RNA, followed by reverse transcription and quantification. U1 and GAPDH were respectively employed as the internal reference controls of nuclear and cytoplasmic fragments. For verifying the results of qRT-PCR, the FISH assay was carried out using Cy-3-labeled circ-PGAP3 and FAM-labeled miR330-3p probes designed by RiboBio (Guangzhou, China) with the Fluorescent in Situ Hybridization Kit (RiboBio). The results were observed using a confocal laser microscope.

\section{Cell Lines and Transfection}

Two TNBC cell lines, MDA-MB-231 and HS598T, were purchased from ATCC and cultured in DMEM medium. Two antisense oligonucleotides (ASOs) targeting the backsplicing site of circ-PGAP3 were synthesized by RiboBio, and were transfected into MDA-MB-231 and HS598T cells using riboFECT ${ }^{\mathrm{TM}} \mathrm{CP}$ (RiboBio) reagent as per the manufacturer's instructions to silence circ-PGAP3. In addition, miR-330-3p mimics and inhibitors purchased from RiboBio were transfected into TNBC cells using riboFECT ${ }^{\mathrm{TM}} \mathrm{CP}$ (RiboBio) reagent to overexpress and inhibit miR-330-3p, respectively.

\section{CCK-8 and Colony Formation Assays}

A total of 1000 transfected MDA-MB-231 and HS598T cells were seeded into 96-well plates added with DMEM complete medium, and cultured for $24 \mathrm{~h}, 48 \mathrm{~h}$ and $72 \mathrm{~h}$, respectively. During this time, the CCK- 8 solution purchased from Dojindo (Kumamoto, Japan) was added to the cells, followed by incubation for $2 \mathrm{~h}$. The absorbance was 
tested using a microplate reader. For the colony formation assay, 500 transfected cells were seeded into 6-well plates and cultured for 2 weeks, and the medium was changed every 2 to 3 days. Then, cells were stained by crystal violet and the number of cell clones was recorded.

\section{Cell Invasion Assay}

A total of 50,000 transfected TNBC cells were seeded into each well of the matrigel-coated upper chamber of 24-well plates, while the lower chamber was added with DMEM complete medium. Then, the 24-well plates were plated in a cell incubator at $37{ }^{\circ} \mathrm{C}$ and cultured for $18 \mathrm{~h}$. The invaded cells were washed and stained by crystal violet, and the results were observed under a light microscope.

\section{RNA Immunoprecipitation (RIP)}

The Magna RIP ${ }^{\mathrm{TM}}$ RNA-binding Protein Immunoprecipitation Kit purchased from Millipore (Schwalbach, Germany) was employed to conduct the RIP assay in accordance with the manufacturer's protocol. In brief, the magnetic beads coated with $8 \mu \mathrm{g}$ specific antibodies against Ago2 (ab186733; Abcam) and normal IgG antibody (Millipore) were incubated with prepared MDA-MB-231 and HS598T cell lysates overnight at $4{ }^{\circ} \mathrm{C}$. After washing by PBST and digestion by proteinase $\mathrm{K}$, the magnetic beads were incubated with TRIzol reagent, followed by RNA extraction and qRT-PCR analysis of circ-PGAP3 enrichment.

\section{RNA Pull-Down Assay}

MDA-MB-231 and HS598T cell lysates were collected and incubated with biotin-labeled circ-PGAP3 probe, or biotin-labeled miR-330-3p mimics were transfected into TNBC cells. After that, cell lysates were incubated with streptavidin-conjugated magnetic beads (Invitrogen) at 25 ${ }^{\circ} \mathrm{C}$ for $3 \mathrm{~h}$. After the beads were attached to the magnetic frame, the lysates were discarded and Trizol was added to extract total RNA, followed by qRT-PCR analysis of circPGAP3 or miRNA enrichment.

\section{Luciferase Reporter Assay}

The wild-type or mutant full-length circ-PGAP3 or Myc 3'-UTR was inserted into pmirGLO vector (Promega, WI, USA). Then, they were co-transfected with control or miR-330-3p mimics into MDA-MB-231 and HS598T cells using riboFECT ${ }^{\text {TM }}$ CP (RiboBio) reagent. After 48 $\mathrm{h}$ of transfection, the Renilla and firefly luciferase activities were detected by the Synergy Mx Multi-Mode Microplate Reader (BioTek, VT, USA).

\section{Western Blot and Immunohistochemistry (IHC)}

The transfected MDA-MB-231 and HS598T cells were treated with RIPA lysis buffer supplemented with protease inhibitor cocktail tablet and phosphatase inhibitor for protein extraction. Afterward, cells were centrifuged at 14,000 rpm for $10 \mathrm{~min}$ at $4{ }^{\circ} \mathrm{C}$. The supernatant was reserved and employed for protein measurement. Then, $20 \mu \mathrm{g}$ of protein was run on $7.5-12.5 \%$ SDS-PAGE gel, and electrotransferred to PVDF membrane. Once blocked with $0.5 \%$ skim milk powder, the membrane was incubated at $4{ }^{\circ} \mathrm{C}$ with Myc primary antibody (ab39688; Abcam). Then, the antiRabbit IgG was added and incubated for $1 \mathrm{~h}$ at room temperature. The Novex ${ }^{\circledR}$ ECL HRP Chemiluminescent Substrate Reagent Kit (Invitrogen) was employed for band visualization. For IHC staining, the paraffin-embedded TNBC patient and xenograft tumor tissues were dewaxed by xylene, blocked by $\mathrm{H}_{2} \mathrm{O}_{2}$ and $\mathrm{BSA}$, incubated with above Myc primary antibody (ab39688; Abcam), and visualized with DAB solution. Lastly, the tissue slices were sealed by neutral gum. The staining result was quantified by the H-score method as previously described. ${ }^{14}$

\section{In vivo Tumourigenesis and Metastasis Assays}

The female athymic BALB/c nude mice aged 4-5 weeks were regularly fed under the standard pathogen-free conditions. Then, $1 \times 10^{7}$ MDA-MB-231 cells were subcutaneously inoculated into the right axillas of mice. One week later, intratumoral injection of ASO-control or ASO-circ-PGAP3\#1 was conducted. At the end of the fifth week, all mice were euthanized and the tumor weight was measured by an electronic scale. Tumor tissues were collected for qRT-PCR analysis and IHC staining. Tail intravenous injection of $1 \times 10^{6}$ MDA-MB-231 cells was carried out for establishing the experimental lung metastasis model. Afterward, ASO-control or ASO-circPGAP3\#1 was injected two to three times a week into the caudal vein for 3 weeks. At the end of the seventh week, $3.0 \mathrm{mg}$ of D-Luciferin was intraperitoneally injected into nude mice, and the lung metastasis nodules were observed using the IVIS@ Lumina II system (Caliper Life Sciences, MA, USA). Then, all mice were euthanized, and the number of lung metastasis nodules was recorded after hematoxylin \& eosin staining. All animal experiment procedures were carried out following the ethical standards under a protocol approved by the Animal Experimental Ethics Committee of Shenzhen Longhua District Central Hospital, and were executed 
conforming to the "Guide for the Care and Use of Laboratory Animals" (revised 1985).

\section{Statistical Analysis}

All of the experiments were repeated three times independently. Student's $t$-test was used for comparison between the two groups. The survival curve was generated by KaplanMeier plot and the difference was analyzed by log rank test. Pearson's correlation coefficient was employed to test the correlation between circ-PGAP3, miR-330-3p and Myc. $P$ value less than 0.05 is statistically different.

\section{Results}

\section{Circ-PGAP3 is Upregulated in TNBC}

\section{Tissues}

A total of 86 paired TNBC and normal tissues were collected for testing circ-PGAP3 expression, and the qRT-PCR results showed that circ-PGAP3 was significantly increased in TNBC tissues in comparison to normal tissues (Figure 1A).
As shown in Table 1, high circ-PGAP3 was closely associated with large tumor size, lymph node metastasis and advanced TNM stage. Importantly, the overall and disease-free survival times of cases with high expression of circ-PGAP3 were both shorter than those of cases with low circ-PGAP3 expression (Figure 1B and C). Then, we treated TNBC cells with RNase $\mathrm{R}$, an exoribonuclease that can degrade almost all linear RNA, but not circRNA. The qRT-PCR results displayed that circ-PGAP3 expression remained unchanged, while linear PGAP3 mRNA was substantially reduced by RNase $\mathrm{R}$ treatment (Figure 1D), suggesting that circ-PGAP3 is actually a circRNA. The results of nucleo-cytoplasmic separation experiments showed that most of circ-PGAP3 was located in the cytoplasm (Figure 1E), and this subcellular localization was also confirmed by FISH assay (Figure 1F).

\section{Circ-PGAP3 Knockdown Inhibits TNBC Cell Proliferation and Invasion}

Due to the upregulation of circ-PGAP3 in TNBC, we designed two ASOs against the back-splicing site of circ-PGAP3 to
A

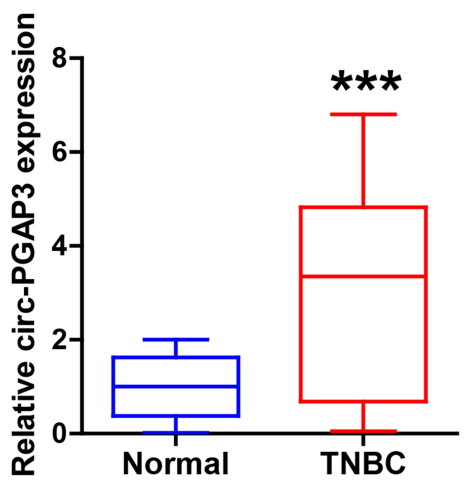

D

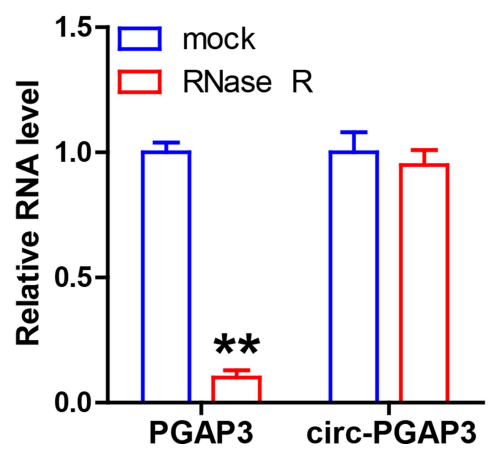

B

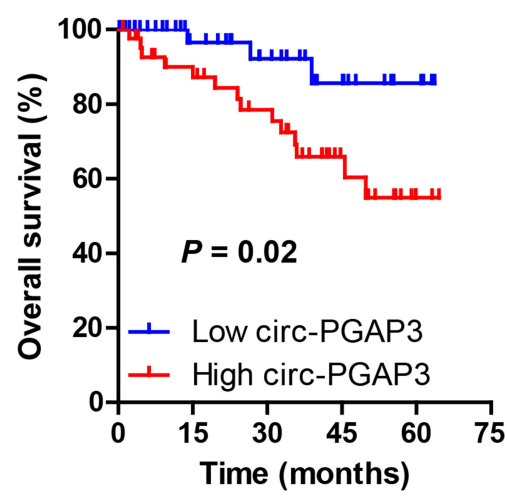

E

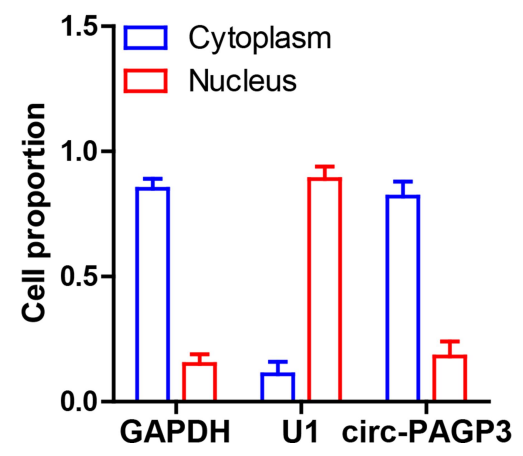

C

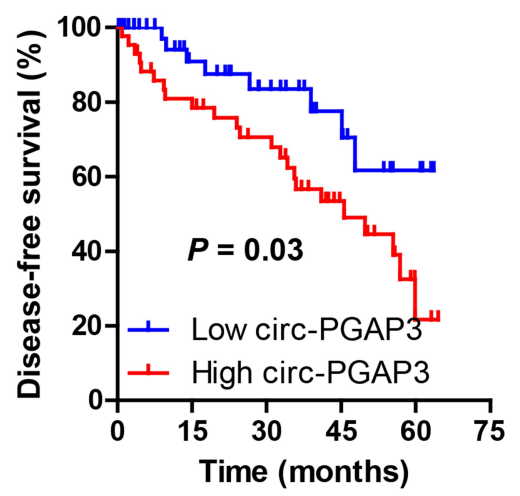

$\mathbf{F}$

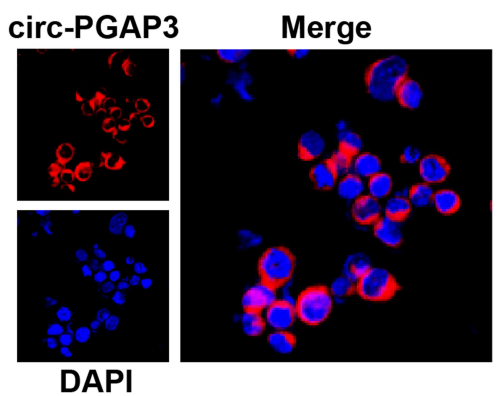

Figure I Circ-PGAP3 is overexpressed in TNBC tissues. (A) qRT-PCR analysis of circ-PGAP3 level in 86 TNBC and matched normal tissues. (B, C) The overall and diseasefree survival curves of patients with low and high circ-PGAP3 expression. (D) After MDA-MB-23I cells were treated with RNase R, the expression of circ-PGAP3 and PGAP3 was detected by qRT-PCR. (E, F) qRT-PCR and FISH testing the location of circ-PGAP3 in MDA-MB-23I cells. The difference between two groups was tested by Student's $t$-test. $* * P<0.01$, $* * * P<0.00$ I. 
Table I Association of Circ-PGAP3 Expression with Clinical Features in 86 Triple-Negative Breast Cancer Patients

\begin{tabular}{|c|c|c|c|c|}
\hline \multirow[t]{2}{*}{ Parameters } & \multirow[t]{2}{*}{$\begin{array}{l}\text { Total } \\
(n=86)\end{array}$} & \multicolumn{2}{|c|}{$\begin{array}{l}\text { Circ-PGAP3 } \\
\text { Expression }\end{array}$} & \multirow[t]{2}{*}{$p$ value } \\
\hline & & $\begin{array}{l}\text { Low } \\
(n=43)\end{array}$ & $\begin{array}{l}\text { High } \\
(n=43)\end{array}$ & \\
\hline \multicolumn{5}{|l|}{ Age (years) } \\
\hline$\leq 40$ & 20 & 8 & 12 & 0.307 \\
\hline$>40$ & 66 & 35 & 31 & \\
\hline \multicolumn{5}{|l|}{ Menopause } \\
\hline Yes & 34 & 18 & 16 & 0.659 \\
\hline No & 52 & 25 & 27 & \\
\hline \multicolumn{5}{|l|}{ Tumor size $(\mathrm{cm})$} \\
\hline$\leq 2$ & 34 & 23 & 11 & 0.008 \\
\hline$>2$ & 52 & 20 & 32 & \\
\hline \multicolumn{5}{|l|}{$\begin{array}{l}\text { Lymph node } \\
\text { metastasis }\end{array}$} \\
\hline Negative & 31 & 24 & 7 & 0.000 \\
\hline Positive & 55 & 19 & 36 & \\
\hline \multicolumn{5}{|l|}{ TNM stage } \\
\hline 1 & 21 & 16 & 5 & 0.001 \\
\hline II & 42 & 22 & 20 & \\
\hline III & 23 & 5 & 18 & \\
\hline
\end{tabular}

silence circ-PGAP3 in MDA-MB-231 and HS598T cells (Figure 2A). As shown in Figure 2B, these two ASOs could effectively decrease the circ-PGAP3 level, but had no effect on the linear PGAP3 level. The CCK-8 results showed that knockdown of circ-PGAP3 evidently subsided cell viability (Figure $2 \mathrm{C})$. Also, the clone ability of circ-PGAP3-depleted cells was significantly weaker than that of control cells (Figure 2D). The invasion assays showed that fewer TNBC cells passed through the artificial matrigel after silencing of circ-PGAP3 (Figure $2 \mathrm{E})$. These data indicate that circ-PGAP3 is a tumor-promoting circRNA in TNBC.

\section{Circ-PGAP3 Acts as a Sponge of miR-330-3p in TNBC Cells}

In light of the cytoplasmic location of circ-PGAP3, we speculated that it might act as a miRNA sponge. We first performed the RIP assay with Ago2 antibody, and the results showed that large numbers of circ-PGAP3 were immunoprecipitated by Ago2 (Figure 3A). Then, we analyzed three online tools (CircInteractome, CircBank and CircNet) to search for which miRNAs might interact with circ-PGAP3. Four miRNAs were simultaneously predicted by these three databases, namely miR-330-3p, miR-384, miR-924 and miR-1227 (Figure 3B).
Afterward, the biotinylated circ-PGAP3 probe was synthesized and the RNA pull-down assay coupled qRT-PCR analysis verified that it could efficaciously enrich circ-PGAP3, but not linear PGAP3 (Figure 3C). As shown in Figure 3D, only miR-330-3p was pulled down by circ-PGAP3 probe in both MDA-MB-231 and HS598T cells. The complementary sequence of circ-PGAP3 and miR-330-3p is displayed in Figure 3E, and we mutated the circ-PGAP3 sequence and performed the luciferase reporter assay. The results showed that miR-330-3p overexpression significantly reduced the luciferase activity of wild-type circ-PGAP3 reporter, whereas it did not alter that of mutant reporter (Figure 3F). Reciprocally, circ-PGAP3 was also abundantly enriched by biotin-labeled miR-330-3p probe (Figure 3G). Knockdown of circ-PGAP3 resulted in significant upregulation of miR-330-3p (Figure $3 \mathrm{H}$ ); in comparison to normal tissues, miR-330-3p was notably decreased in TNBC tissues (Figure 3I); and its expression was inversely correlated with circ-PGAP3 expression $(\mathrm{r}=$ -0.745 ) (Figure 3J). Besides, the co-location of circ-PGAP3 and miR-330-3p was observed in TNBC cells, as shown by FISH assay (Figure 3K).

\section{Circ-PGAP3 Regulates the miR-330-3p/ Myc Axis in TNBC Cells}

Through analyzing the miRwalk database, we found that the well-known proto-oncogene Myc might be the downstream target of miR-330-3p, and we then mutated the binding sequence between miR-330-3p and Myc 3'-UTR (Figure $4 \mathrm{~A}$ ), and conducted the luciferase reporter assay. As shown in Figure 4B, miR-330-3p overexpression resulted in a significant decrease in luciferase activity of wild-type Myc 3 '-UTR vector, while that of the mutated vector remained unchanged. Importantly, the expression of Myc mRNA was markedly increased by miR-330-3p knockdown, but this effect completely disappeared after circ-PGAP3 knockdown (Figure 4C). Consistently, silencing of circ-PGAP3 led to a significant decrease in Myc protein level in both MDA-MB-231 and HS598T cells, and miR-330-3p knockdown partially rescued this downregulation (Figure 4D). Then, we conducted IHC staining of Myc in TNBC and normal tissues, and the results displayed that Myc protein was significantly overexpressed in TNBC (Figure 4E), and its expression was strongly positively correlated with circ-PGAP3 expression ( $\mathrm{r}=0.781$ ) (Figure 4F). Functionally, the declined cell viability and invasion induced by circ-PGAP3 knockdown were substantially blocked after miR-330-3p knockdown or Myc overexpression (Figure 4G and $\mathrm{H})$. 
A

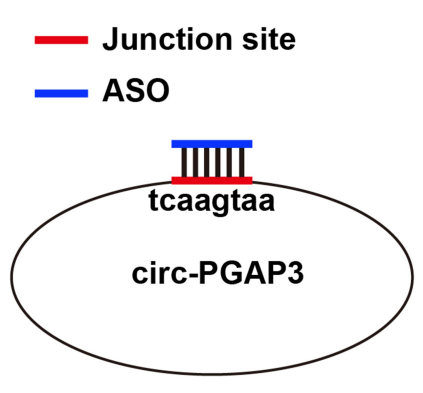

C

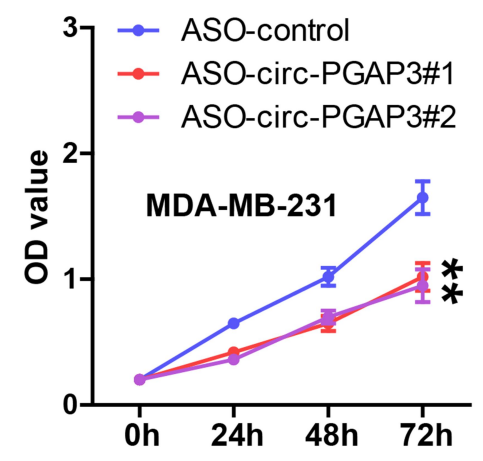

B
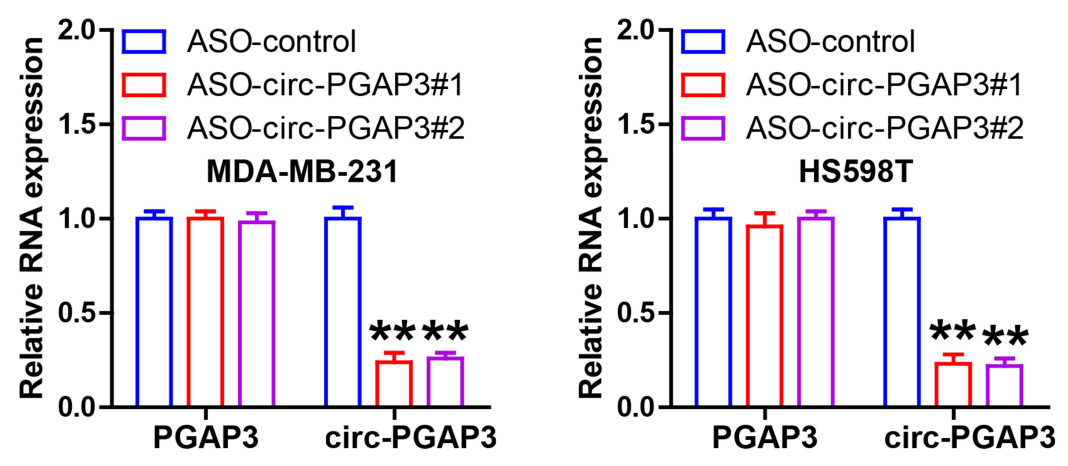

D
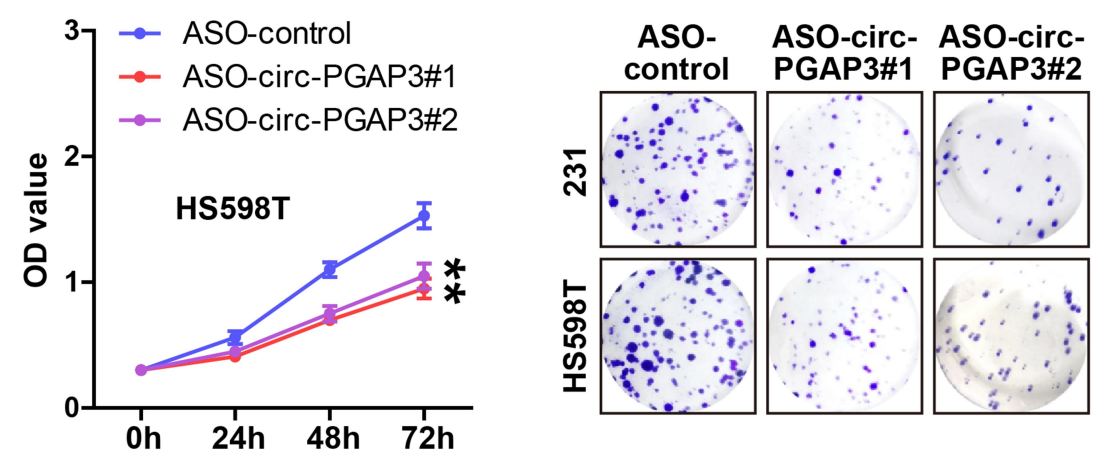

$E$
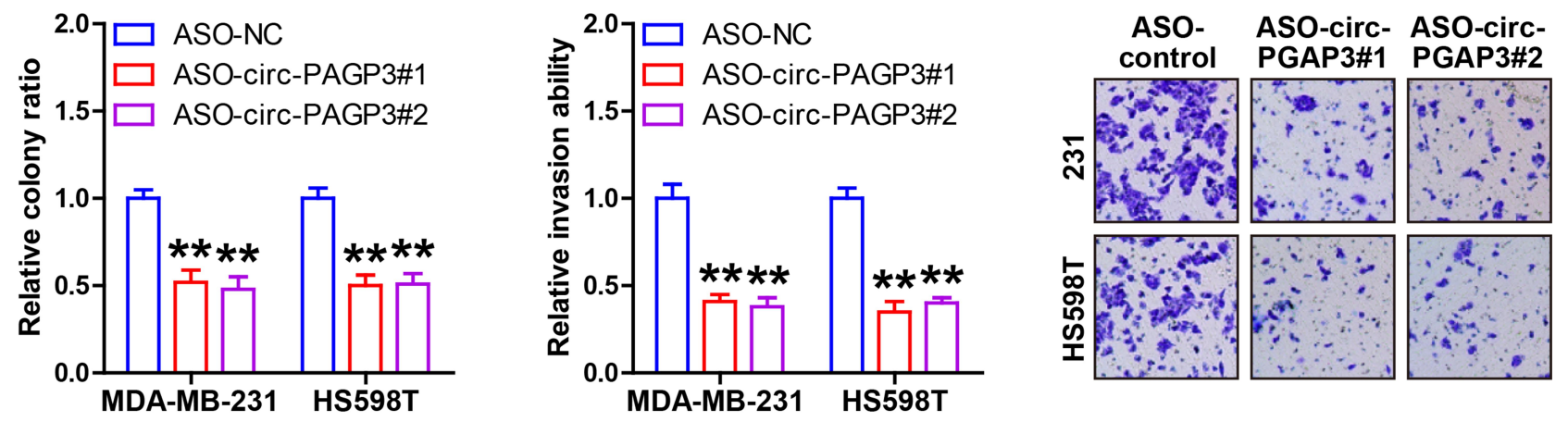

Figure 2 Inhibition of circ-PGAP3 reduces TNBC cell proliferation and invasion. (A) Cartoon showing the designed ASO targeting the junction site of circ-PGAP3 silencing circ-PGAP3. (B) qRT-PCR analysis of circ-PGAP3 and PGAP3 expression after transfection with ASO targeting circ-PGAP3. (C-E) CCK-8, colony formation and Transwell invasion assays testing cell viability, colony ability and invasion after circ-PGAP3 knockdown in the indicated two TNBC cell lines. The difference between two groups was tested by Student's $t$-test. $* * P<0.01$.

\section{Knockdown of Circ-PGAP3 Represses Tumor Growth and Metastasis in vivo}

Lastly, we tested whether circ-PGAP3 functioned in vivo through establishing the xenograft tumor and experimental lung metastasis models. The results showed that the tumor volume and weight of nude mice bearing circ-PGAP3silenced MDA-MB-231 cells were significantly less than those of mice bearing control cells (Figure 5A-C). Also, the qRT-PCR analysis showed that miR-330-3p was evidently increased in circ-PGAP3-depleted tumor tissues, whereas linear PGAP3 expression was not affected (Figure 5D). Likewise, fewer Myc-positive cells were observed in circPGAP3-depleted group as compared to the control group (Figure 5E). The results of the experimental lung metastasis 
A

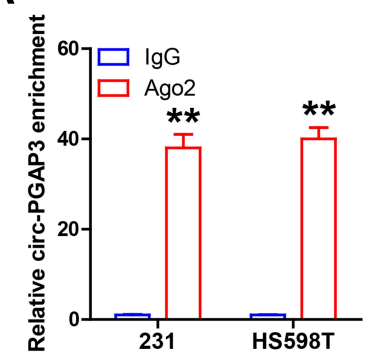

D
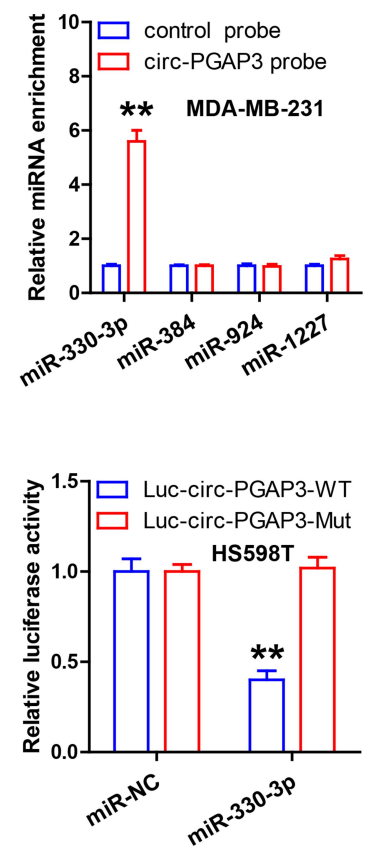

I

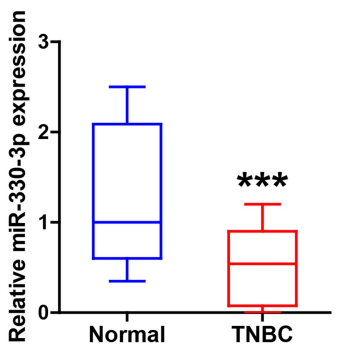

B

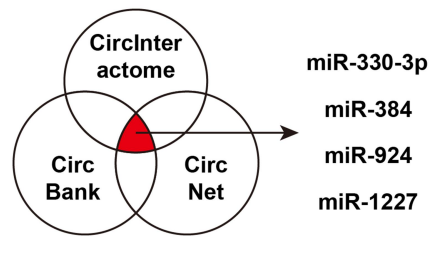

C

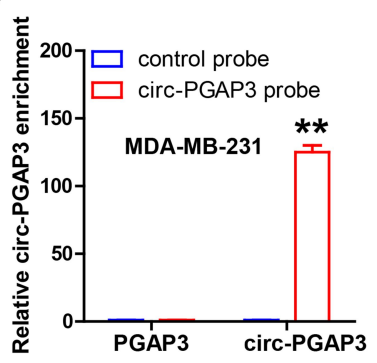

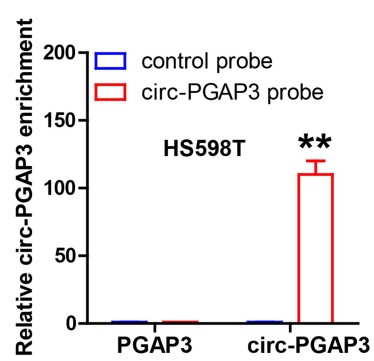

E

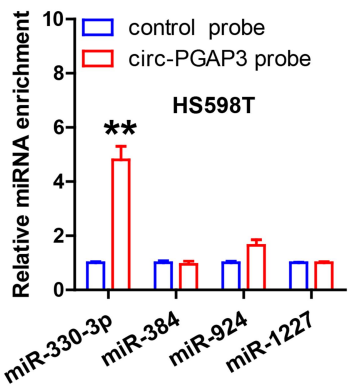

F circ-PGAP3 WT

5-AAGGUGUCAGUUCCGGCUUUGA-3 miR-330-3p $\quad\||\||$ 3'-GAGACGUCCGGCACACGAAACG-5 circ-PGAP3 Mut

5 -AAGGUGUCAGUUCCGGCUHUA-3' CGAAAC

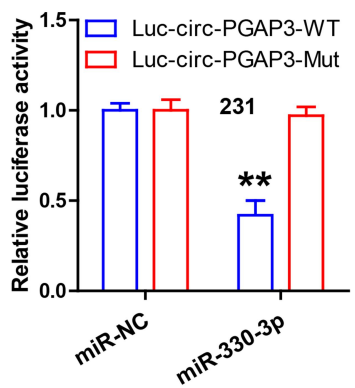

G

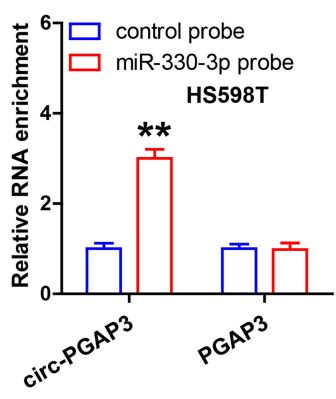

H

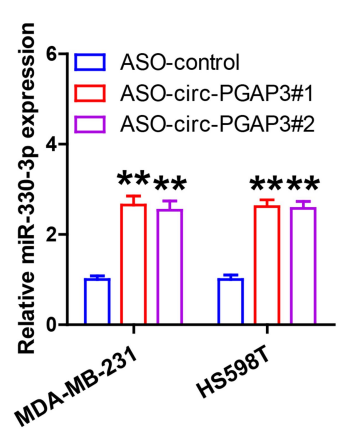

J
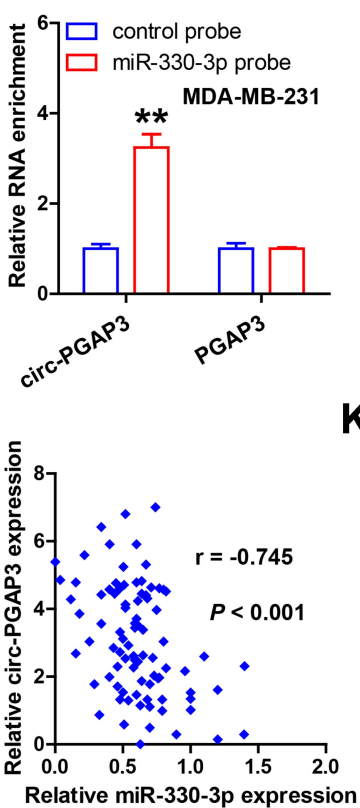

$\mathbf{K}$

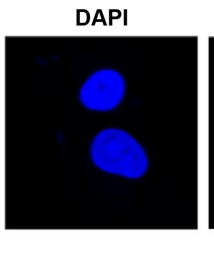

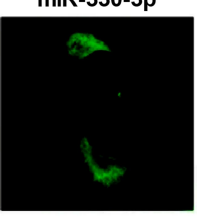

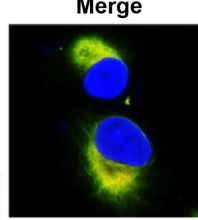

Figure 3 miR-330-3p is the downstream target of circ-PGAP3 in TNBC cells. (A) RIP assay using Ago2 antibody detecting the enrichment of circ-PGAP3 by Ago2. (B) The four miRNAs predicted by the indicated three databases. (C) qRT-PCR analysis following RNA pull-down assay verifying the availability of the designed biotin-labeled probes. (D) qRT-PCR analysis following RNA pull-down assay testing the miRNAs bound by circ-PGAP3. (E) The complementary sequences of circ-PGAP3 and miR-330-3p. (F) The luciferase reporter assay in the indicated two TNBC cells co-transfected with wild-type or mutant circ-PGAP3 vector and control or miR-330-3P mimics. (H) qRT-PCR analysis of miR-330-3p expression after circ-PGAP3 knockdown. (I) qRT-PCR analysis of miR-330-3p expression in TNBC and normal tissues. (J) The correlation between circ-PGAP3 and miR-330-3p expression in TNBC tissues. (K) FISH assay detecting the co-location between circ-PGAP3 and miR-330-3p. The difference between two groups was tested by Student's $t$-test. $* * P<0.01$, $* * * P<0.001$.

model showed that the average number of lung metastatic nodules in the control group was 32 , while the average number in the circ-PGAP3-depleted group was only 10 (Figure 5F). These data suggest that circ-PGAP3 is also an oncogenic circRNA in vivo.

\section{Discussion}

Emerging evidence indicates that circRNA is a critical regulator in human cell fate. ${ }^{15}$ In the present study, we for the first time explored the expression and role of circ-PGAP3 in TNBC, an aggressive and fatal disease. 
A

MYC 3'-UTR WT

5 -UAUUUAaguacaUUUgCUUUUA-3' miR-330-3p

3`-GAGACGUCCGGCACACGAAACG-5

MYC 3'-UTR Mut

5 -UAUUUAAGUACAUUGGCÜUA-3' ACGAAA

C
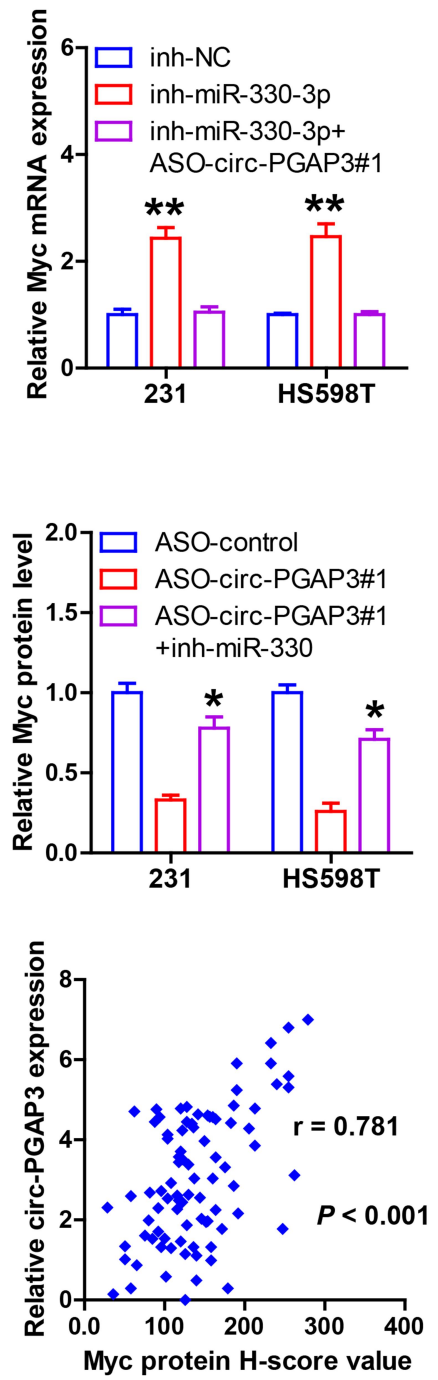

B
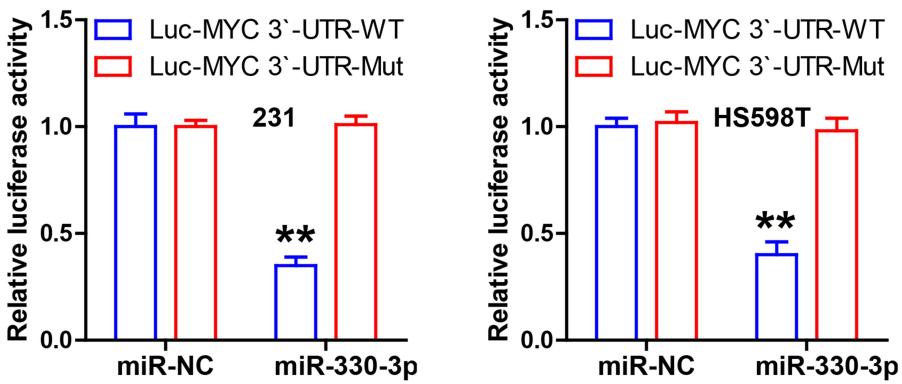

D

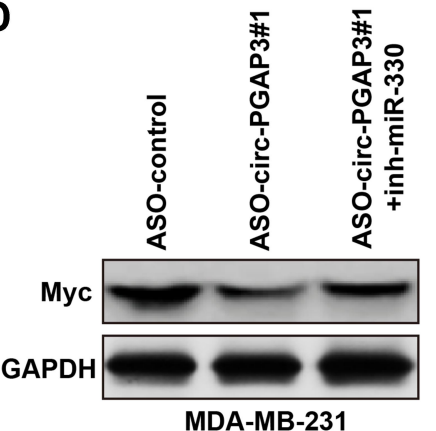

E
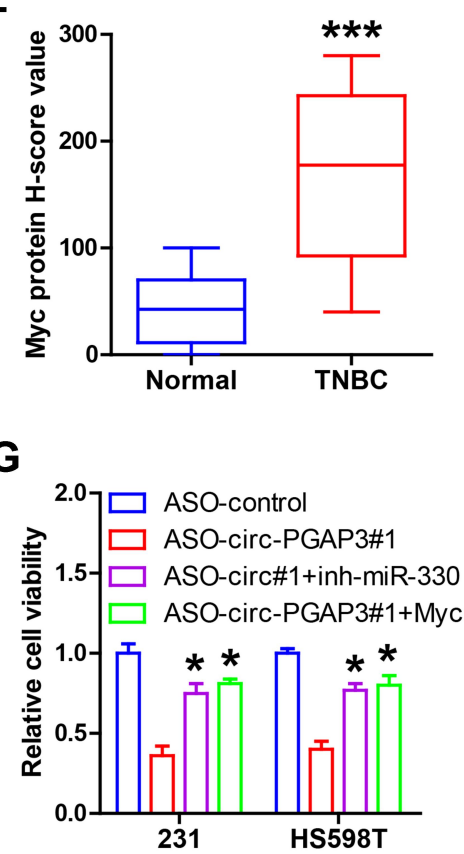

$\mathbf{F}$

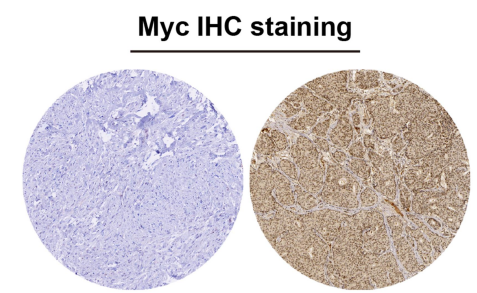

Low circ-PGAP3 High circ-PGAP3

H

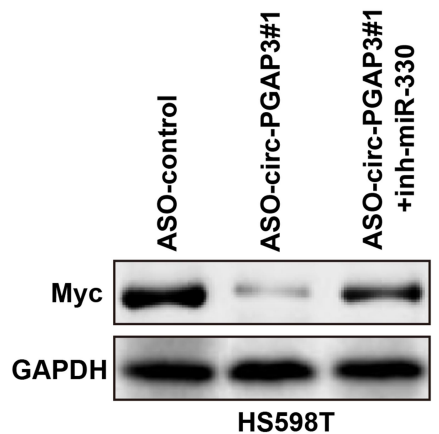

HS598T

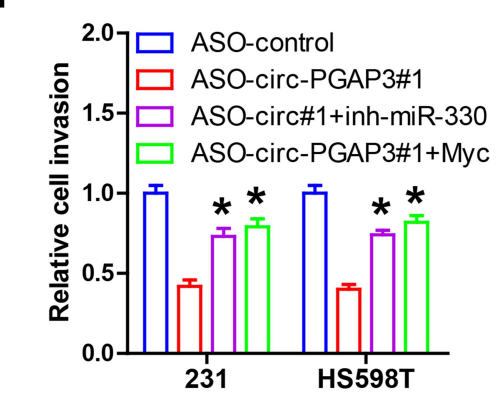

Figure 4 Identification of the circ-PGAP3/miR-330-3p/Myc axis in TNBC cells. (A) The complementary sequences of miR-330-3p and Myc 3'-UTR. (B) The luciferase reporter assay in the indicated two TNBC cells co-transfected with wild-type or mutant Myc 3'-UTR vector and control or miR-330-3p mimics. (C) qRT-PCR analysis of Myc mRNA expression in miR-330-3p-depleted TNBC cells transfected with ASO against circ-PGAP3. (D) Western blot analyzing the protein level of Myc in circ-PGAP3depleted TNBC cells transfected with miR-330-3p inhibitors. (E) IHC staining of Myc in TNBC and normal tissues. (F) High Myc protein level was positively correlated with high circ-PGAP3 expression. (G-H) Cell viability and invasion were tested in circ-PGAP3-depleted TNBC cells transfected with miR-330-3p inhibitors or Myc expression plasmid. The difference between two groups was tested by Student's $t$-test. $* P<0.05, * * P<0.01, * * * P<0.00 \mathrm{I}$.

High circ-PGAP3 expression was observed in TNBC tissues, which was linked to malignant clinical features and poor outcome. Knockdown of circ-PGAP3 inhibits
TNBC cell growth and invasion both in vitro and in vivo. Mechanism research revealed that circ-PGAP3 was a cytoplasmic circRNA and served as a sponge of 


\section{A}

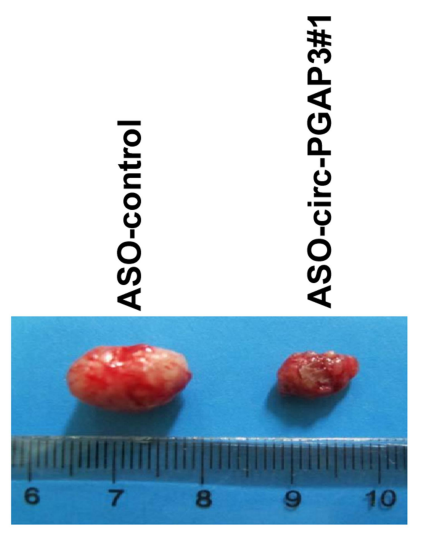

D

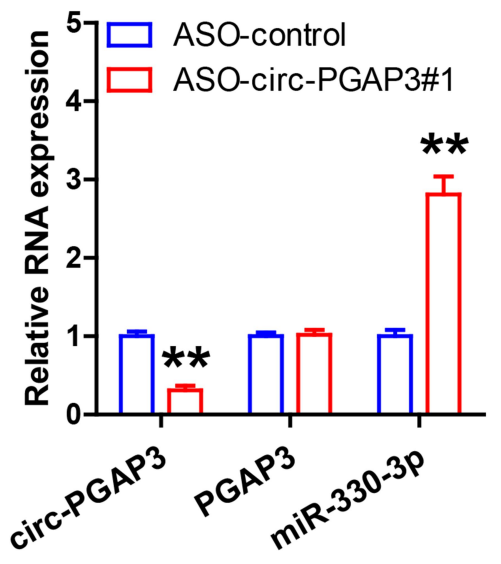

F

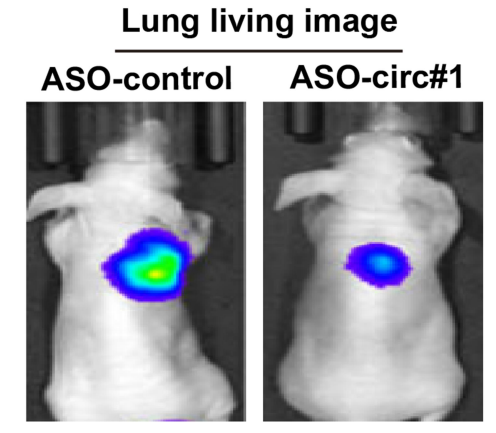

B

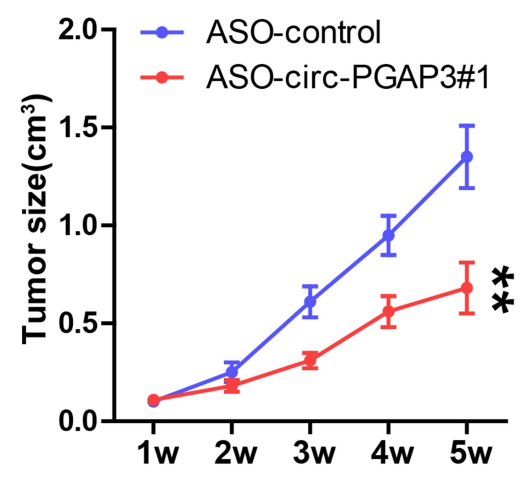

E

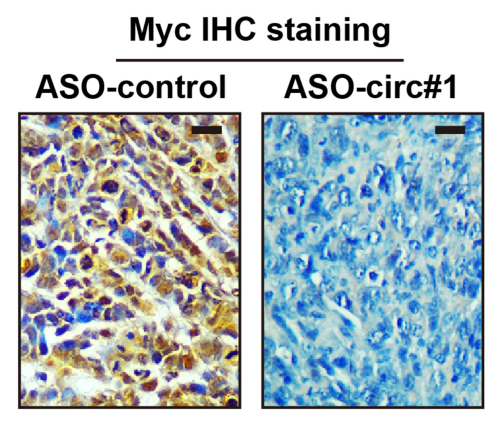

C

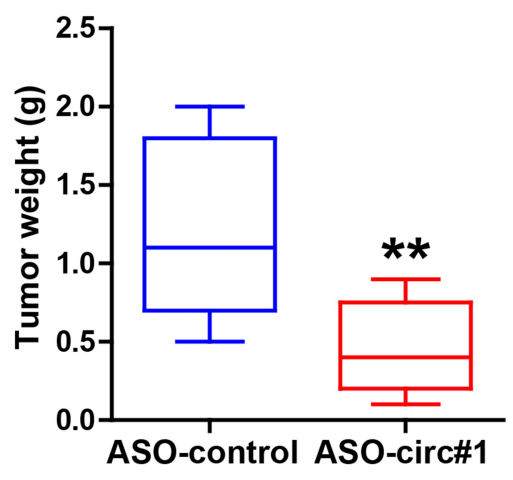

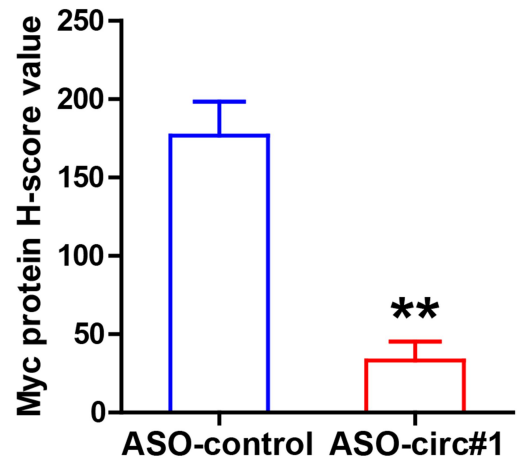
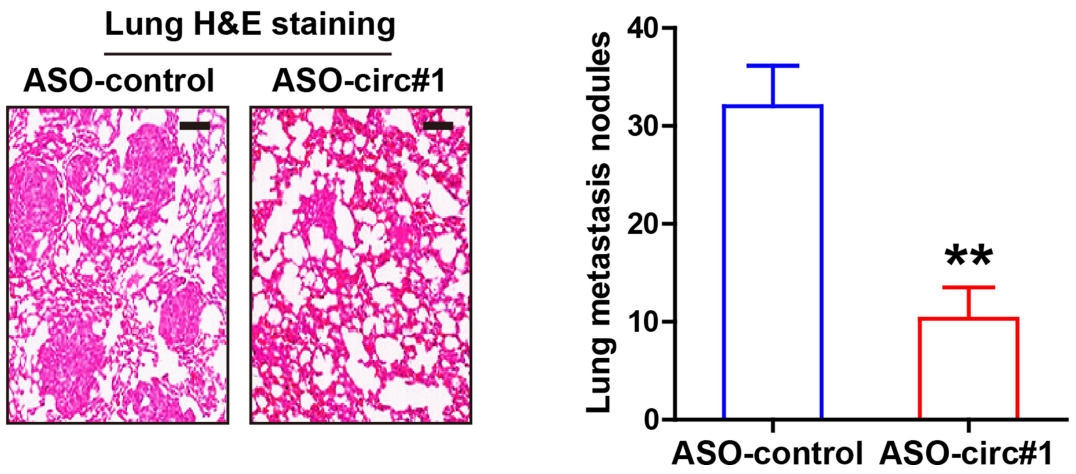

Figure 5 Knockdown of circ-PGAP3 inhibits in vivo TNBC cell growth and invasion. (A-C) Tumor image, volume and weight of nude mice bearing control and circ-PGAP3depleted MDA-MB-23I cells ( $n=5$ per group). (D) qRT-PCR analysis of circ-PGAP3, PGAP3 and miR-330-3p expression in the indicated two groups. (E) IHC staining of Myc in tumor tissues of the indicated two groups. (F) IVIS@ Lumina II system and H\&E staining evaluating lung metastasis in the indicated two groups ( $\mathrm{n}=5$ per group). Scale bar $=25 \mu \mathrm{m}$. The difference between two groups was tested by Student's $t$-test. $* * P<0.01$.

miR-330-3p to increase Myc expression. The regulatory network of circ-PGAP3/miR-330-3p/Myc was also confirmed in TNBC tissues and a xenograft tumor model. Thus, our data provide new evidence for the biological implication of circRNA in human cancer, and also uncover the pivotal tumor-driving role of circ-PGAP3 in TNBC.

To date, the field of circRNA research has attracted a lot of attention. In terms of function, recent studies have shown that circRNA is rich in miRNA binding sites, which 
acts as a miRNA molecular sponge in human cells, thereby antagonizing the inhibition of miRNA on its target genes and increasing their expression levels. ${ }^{16}$ This mechanism of action is called a competitive endogenous RNA (ceRNA) mechanism. ${ }^{17,18}$ Accumulated evidence shows that circRNA plays an important regulatory role in human cancer by serving as a ceRNA. ${ }^{19}$ For example, circPTN was reported to be elevated in hepatocellular carcinoma and promoted tumorigenesis and progression via the sponge activity on miR-326 and increasing the level of ERBB/PI3K. ${ }^{20}$ Circ-AMOTL1L was downregulated in human prostate cancer, and could directly interact with miR-193a-5p to elevate a subset of the cadherin superfamily members, repressing tumor growth. ${ }^{21}$ Circ-HIPK3 was shown as an oncogene that abundantly binds to miR-7 and increases miR-7 targets in human colorectal cancer. ${ }^{22}$ These studies suggest that dysregulation of the ceRNA network of circRNA may be responsible for cancer occurrence, development and progression.

Since mature miRNA is located in the cytoplasm and is assembled into RNA-induced silencing complex by Ago2 protein, ${ }^{23}$ only cytoplasmic circRNA can act as a miRNA molecular sponge and bind to Ago2. Herein, we found that circ-PGAP3 was a cytoplasmic circRNA and abundantly enriched by Ago2 antibody, hinting that it may be a ceRNA absorbing miRNA. Through the bioinformatics analysis coupled RNA pull-down assay, we found that miR330-3p was the downstream miRNA of circ-PGAP3, in which circ-PGAP3 inhibited miR-330-3p activity to increase the expression of Myc, a well-documented oncoprotein that binds to gene promoter, transcriptionally regulating gene expression. ${ }^{24,25}$ Importantly, high circ-PGAP3 was closely linked to low miR-330-3p and high Myc expression in TNBC tissues, and the attenuated cell malignant phenotype caused by circ-PGAP3 silencing was effectively abolished by miR-330-3p inhibition or exogenous Myc expression, suggesting that the ceRNA network of circ-PGAP3/miR-330-3p/Myc indeed exists in TNBC. It is of great interest to explore which factors are responsible for the upregulation of circ-PGAP3 in TNBC (such as some RNA binding proteins affecting the cyclization of circPGAP3, or directly transcriptional regulation by Myc forming a positive feedback loop).

All in all, our findings clearly demonstrate that circPGAP3 is a carcinogenic circRNA in TNBC, which promotes TNBC growth and metastasis via acting as a miRNA sponge. Targeting of circ-PGAP3 may be a promising treatment for TNBC patients.

\section{Disclosure}

The authors declare no conflicts of interest for this work.

\section{References}

1. Metzger-Filho O, Tutt A, de Azambuja E, et al. Dissecting the heterogeneity of triple-negative breast cancer. $J$ Clin Oncol. 2012;30(15):1879-1887. doi:10.1200/JCO.2011.38.2010

2. Criscitiello C, Azim HJ, Schouten PC, Linn SC, Sotiriou C. Understanding the biology of triple-negative breast cancer. Ann Oncol. 2012;23(Suppl 6):vi13-8. doi:10.1093/annonc/mds188

3. Yin L, Duan JJ, Bian XW, Yu SC. Triple-negative breast cancer molecular subtyping and treatment progress. Breast Cancer Res. 2020;22(1):61. doi:10.1186/s13058-020-01296-5

4. Yu K, Rohr J, Liu Y, et al. Progress in triple negative breast carcinoma pathophysiology: potential therapeutic targets. Pathol Res Pract. 2020;216(4):152874. doi:10.1016/j.prp.2020.152874

5. Garrido-Castro AC, Lin NU, Polyak K. Insights into molecular classifications of triple-negative breast cancer: improving patient selection for treatment. Cancer Discov. 2019;9(2):176-198. doi:10.1158/2159-8290.CD-18-1177

6. Bergin A, Loi S. Triple-negative breast cancer: recent treatment advances. F1000Res. 2019;8:1342. doi:10.12688/f1000research.18888.1

7. Patop IL, Wust S, Kadener S. Past, present, and future of circRNAs. EMBO J. 2019; 100836.

8. Wilusz JE. A 360 degrees view of circular RNAs: from biogenesis to functions. Wiley Interdiscip Rev RNA. 2018;9(4):e1478.

9. Hentze MW, Preiss T. Circular RNAs: splicing's enigma variations. EMBO J. 2013;32(7):923-925. doi:10.1038/emboj.2013.53

10. Kristensen LS, Andersen MS, Stagsted L, Ebbesen KK, Hansen TB, Kjems J. The biogenesis, biology and characterization of circular RNAs. Nat Rev Genet. 2019;20(11):675-691. doi:10.1038/s41576019-0158-7

11. Tang Q, Hann SS. Biological roles and mechanisms of circular RNA in human cancers. Onco Targets Ther. 2020;13:2067-2092. doi:10.2147/OTT.S233672

12. Bach DH, Lee SK, Sood AK. Circular RNAs in cancer. Mol Ther Nucleic Acids. 2019;16:118-129. doi:10.1016/j.omtn.2019.02.005

13. Croce CM. Genetics: are circRNAs involved in cancer pathogenesis? Nat Rev Clin Oncol. 2016;13(11):658.

14. Zeng K, He B, Yang BB, et al. The pro-metastasis effect of circANKS1B in breast cancer. Mol Cancer. 2018;17(1):160. doi:10.1186/s12943-018-0914-x

15. Qu S, Zhong Y, Shang R, et al. The emerging landscape of circular RNA in life processes. RNA Biol. 2017;14(8):992-999. doi:10.1080/ 15476286.2016.1220473

16. Bak RO, Mikkelsen JG. miRNA sponges: soaking up miRNAs for regulation of gene expression. Wiley Interdiscip Rev RNA. 2014;5 (3):317-333. doi:10.1002/wrna.1213

17. Tay Y, Rinn J, Pandolfi PP. The multilayered complexity of ceRNA crosstalk and competition. Nature. 2014;505(7483):344-352. doi:10.1038/nature12986

18. Thomson DW, Dinger ME. Endogenous microRNA sponges: evidence and controversy. Nat Rev Genet. 2016;17(5):272-283.

19. Zhong Y, Du Y, Yang X, et al. Circular RNAs function as ceRNAs to regulate and control human cancer progression. Mol Cancer. 2018;17 (1):79.

20. Jia B, Yin X, Wang Y, et al. CircRNA-PTN sponges miR-326 to promote proliferation in hepatocellular carcinoma. Onco Targets Ther. 2020;13:4893-4903. doi:10.2147/OTT.S251300

21. Yang Z, Qu CB, Zhang Y, et al. Dysregulation of p53-RBM25mediated circAMOTL1L biogenesis contributes to prostate cancer progression through the circAMOTL1L-miR-193a-5p-Pcdha pathway. Oncogene. 2019;38(14):2516-2532. doi:10.1038/s41388018-0602-8 
22. Zeng K, Chen X, Xu M, et al. CircHIPK3 promotes colorectal cancer growth and metastasis by sponging miR-7. Cell Death Dis. 2018;9 (4):417. doi:10.1038/s41419-018-0454-8

23. Gurtan AM, Sharp PA. The role of miRNAs in regulating gene expression networks. $J$ Mol Biol. 2013;425(19):3582-3600. doi:10.1016/j.jmb.2013.03.007
24. Collum RG, Alt FW. Are myc proteins transcription factors? Cancer Cells. 1990;2(3):69-75.

25. Dang CV, Resar LM, Emison E, et al. Function of the c-Myc oncogenic transcription factor. Exp Cell Res. 1999;253(1):63-77. doi:10.1006/excr.1999.4686

\section{Publish your work in this journal}

OncoTargets and Therapy is an international, peer-reviewed, open access journal focusing on the pathological basis of all cancers, potential targets for therapy and treatment protocols employed to improve the management of cancer patients. The journal also focuses on the impact of management programs and new therapeutic

Submit your manuscript here: https://www.dovepress.com/oncotargets-and-therapy-journal agents and protocols on patient perspectives such as quality of life, adherence and satisfaction. The manuscript management system is completely online and includes a very quick and fair peer-review system, which is all easy to use. Visit http://www.dovepress.com/ testimonials.php to read real quotes from published authors. 